\title{
INITIATIVEN DER AUSTRIA WIRTSCHAFTSSERVICE GMBH ZUR ANKURBELUNG DES ÖSTERREICHISCHEN RISIKOKAPITALMARKTS
}

\author{
HELMUT GASSLER
}

\section{EINLEITUNG}

$\mathrm{I}$ n internationalen Benchmarks scheint Österreich notorisch in den hinteren Rängen bezüglich der Qualität und Dynamik seines Risikokapitalmarkts auf. Dabei ist Risikokapital nicht zuletzt für eine dynamische innovative Gründungs- bzw. Startup-Szene von enormer Bedeutung, da für diese Unternehmenstypen klassische Bankkredite aufgrund inhärenter Probleme als Finanzierungsform kaum in Frage kommen. Die (derzeit noch) aktuelle FTI-Strategie der österreichischen Bundesregierung aus dem Jahr 2011 formuliert deshalb auch explizit das Ziel, die österreichische Startup-Szene anzukurbeln. Letztlich sollen damit die in der Literatur immer wieder betonten positiven Effekte derartiger Gründungen (z.B. Beitrag zum technologischen Wandel, Beschäftigungswirkung Wachstumswirkung, Wettbewerbswirkung) forciert werden

In Österreich existiert ein umfangreiches und differenziertes Gründungsförderungssystem, das auf unterschiedlichen, sich komplementär ergänzenden Maßnahmen und Instrumenten beruht, die von Awareness-, Beratungs- und Infrastrukturmaßnahmen bis hin zu direkten monetären Unterstützungen (in verschiedensten instrumentellen Ausprägungen) reichen und dessen institutionelle Säulen die beiden großen Förderagenturen des Bundes, nämlich die Austria Wirtschaftsservice $\mathrm{GmbH}$ (aws) sowie die Forschungsförderungsgesellschaft (FFG), bilden. Dieses Fördersystem findet auch bei internationalen Beobachtern bzw. Organisationen (u.a. OECD) regelmäßig Anerkennung.

Allerdings ist die öffentliche Förderung von solchen Unternehmensgründungen lediglich eine Seite der Medaille, denn nach der Gründung und dem erstem Aufbau des Unternehmens folgt eine weitere kritische Phase der Unternehmensentwicklung, nämlich die Skalierung der Geschäftsidee. Für diese Phase wird eine entsprechende Kapitalausstattung benötigt, die vom Umfang her gerade bei (kapitalintensiveren) technologieorientierten Gründungen (insbesondere in Bereichen wie den Life Sciences, der Medizintechnik oder auch der Umwelttechnik) üblicherweise nicht mehr mit öffentlichen Förderungen (allein) abzudecken ist. Gerade diese besonders technologieorientierten Geschäftsfelder sind jedoch aufgrund ihres Innovationssprungs mit hohem Risiko und Unsicherheit behaftet. In Verein mit anderen Ursachen (z.B. fehlendem track record, kaum vorhandene liquidierbare Assets im Falle des Scheiterns, Informationsasymmetrien etc.) führt dies dazu, dass eine Fremdkapitalfi- nanzierung mittels Bankenkrediten kaum bis gar nicht möglich ist.

Eine Möglichkeit, diese chronische Lücke der Frühphasenfinanzierung zu schließen stellt der private (Risiko-)Kapitalmarkt dar, wobei Risikokapital in Form von institutionellen Risikokapitalfonds auch von privaten Kapitalgebern (Business Angels) zur Verfügung gestellt werden kann. Zusätzlich gewann in den letzten Jahren - wenn auch auf (noch) niedrigem Niveau -sogenanntes Crowdinvesting auch in Österreich etwas an Bedeutung.

Der Risikokapitalmarkt stellt also im Idealfall innovativen, erfolgversprechenden Unternehmen nach entsprechender Prüfung der Aussichten der Geschäftsidee gegen eine Beteiligung am Unternehmen Kapital (und meist auch Knowhow) zur Verfügung, wobei die Absicht verfolgt wird, die Beteiligung nach einer bestimmten Zeitspanne (üblicherweise maximal zehn Jahre) mit entsprechendem Gewinn wieder zu veräußern.

\section{ZUR AKTUELLEN ENTWICKLUNG DES ÖSTERREICHISCHEN RISIKOKAPITALMARKTS}

In der Literatur wird eine systemische Betrachtung der Gründungslandschaft sowie seiner institutionellen Rahmenbedingungen (Schramm, 2006; OECD, 2014; Acs et al., 2014) betont: „Remember that entrepreneurial activity does not exist in a vacuum" (Lerner, 2010, S. 261). Als „entrepreneurial ecosystem“ kann dabei die Gesamtheit der Individuen und Institutionen sowie deren Interaktionen, die in verschiedenster Art und Weise Ausmaß und Struktur der Gründungsaktivitäten bestimmen, verstanden werden (siehe Abbildung 1)

Neben den (potentiellen) Gründerlnnen selbst zählen Inkubatoren wie z.B. Universitäten und sonstige Forschungseinrichtungen, Förderinstitutionen, regulative bzw. administrative Rahmenbedingungen, Business Angels und institutionelle Risikokapitalgeber (Venture Capital Fonds), einschlägige Interessensvertretungen, spezialisierte Dienstleister (z.B. Steuerberater oder Juristen mit einschlägiger Spezialisierung) zu den Elemente dieses Ökosystems. Zu betonen ist, dass vor allem das Ausmaß und die Qualität der Interaktionen zwischen diesen Akteuren 
bzw. Institutionen die Qualität und Dynamik eines derartigen Ökosystems bestimmt. Viele dieser Interaktionen erfolgen dabei über (auch informelle) personelle Netzwerke, wodurch laufend sogenanntes „Sozialkapital" generiert wird. Für die Ausgestaltung der Politik hat diese systemische Betrachtung zur Folge, dass die einzelnen Politikelemente (Gründungsförderung, Risikokapitalmarktpolitik, Rahmenbedingungen etc.) optimal aufeinander abgestimmt sein sollten. Fehlt eine Komponente (z.B. Risikokapitalmarkt bzw. -politik) hat das wiederum negative Effekte auf die anderen Komponenten. tion Leader-Ländern (Dänemark, Schweden, Finnland und Deutschland) nachhinkt.

Im Folgenden soll anhand von Daten der European Venture Capital Association (kurz EVCA, heute Invest Europe') Struktur und Dynamik des Risikokapitalmarkts in Österreich etwas näher charakterisiert werden. Abbildung 2 zeigt die in Österreich investierten Venture Capital Summen einerseits als auch die Zahl der Beteiligungsfälle in entsprechende Portfoliounternehmen². Die jährlichen Investitionssummen belaufen sich im betrachteten Zeitraum (2007 bis 2015) auf ein Minimum von knapp

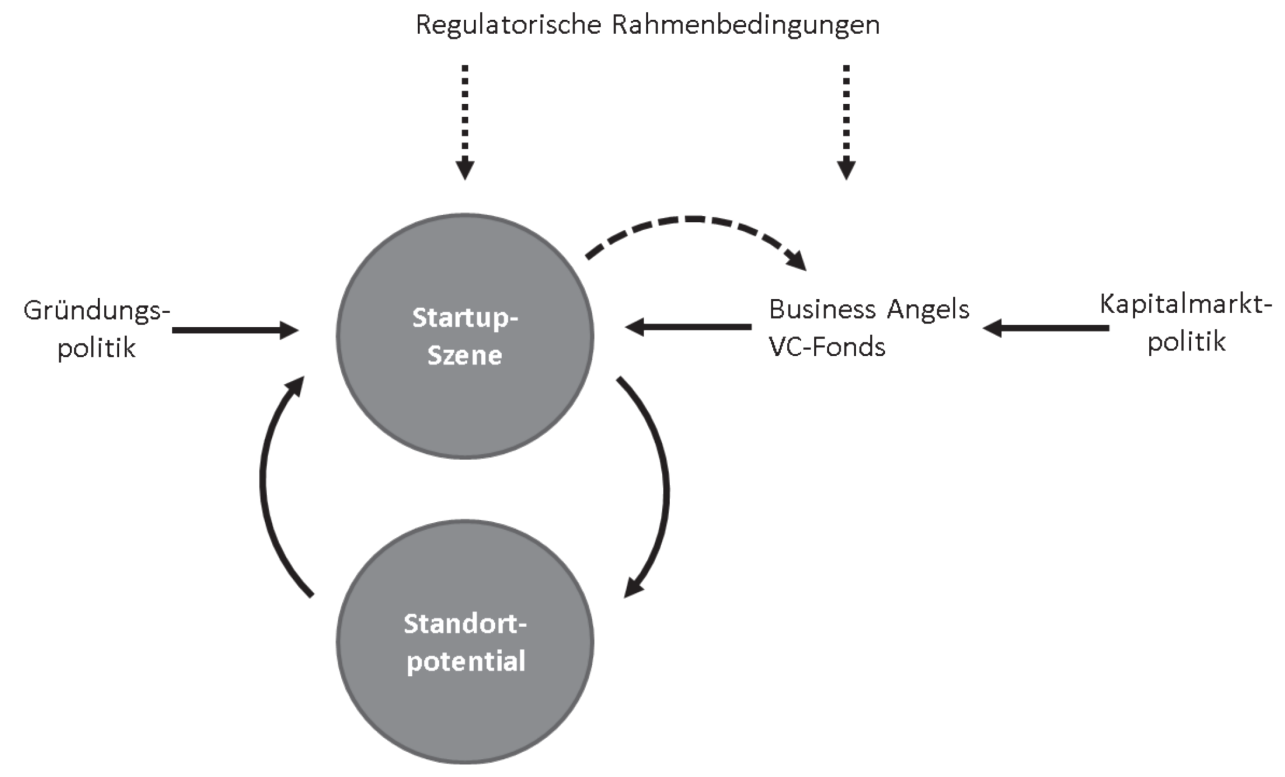

Abbildung 1: Schematische Darstellung eines Gründungs-Ökosystems Quelle: Eigene Darstellung.

Der Risikokapitalmarkt ist in Kontinentaleuropa generell als „junger" Markt anzusehen und befindet sich demnach hier (darunter nicht zuletzt vor allem auch in Österreich) noch in einem frühen Stadium der „infancy”. Gerade auch für Österreich gilt, dass sich der Risikokapitalmarkt auch während vergangener "hypes" (z.B. new economy boom in den späten 1990er Jahre, Konjunkturhausse bis 2007) nur sehr zögerlich dynamisiert hatte. In Folge des Zusammenbruchs dieser jeweiligen Boomphasen ist er dann regelmäßig wieder fast gänzlich ausgetrocknet (z.B. dramatischer Rückgang des Fundraising im Zuge der Finanz- und Wirtschaftskrise im Jahr 2008 und den darauf folgenden Jahren und weitgehendes Aussteigen wichtiger institutioneller Akteure wie z.B. Banken, Versicherungen, Pensionskassen etc.).

Österreich ist somit traditionell durch eine geringe Verfügbarkeit von Risikokapital (gemessen als Anteil am Bruttoinlandsprodukt (BIP)) geprägt, ein Umstand, der auch in die regelmäßige Berichterstattung des European Innovation Scoreboard (EIS) der Europäischen Kommission einfließt und mit dazu beiträgt, dass Österreich gegenüber den Innova- über EUR 40 Mio. im Jahr 2012 bis hin zu einem Maximum von ca. EUR 111 Mio. am aktuellen Rand des Beobachtungszeitraumes, d.h. im Jahr 2015. D.h., dass es jüngst gegenüber den Jahren 2013 bzw. 2014 (jeweils ca. EUR 60 Mio. VC-Investitionen) annähernd eine Verdoppelung gab. Generell zeigt sich bezüglich der Investitionssummen jedoch ein sehr volatiles Bild; die Schwankungen zwischen den einzelnen Jahren sind durchaus erheblich. Anzumerken ist diesbezüglich jedoch, dass diese starken jährlichen Schwankungen gerade aufgrund der Kleinheit und Enge des österreichischen Risikokapitalmarkts zustande kommen. Einige wenige Einzelfälle mit überdurchschnittlichen Investitionsentscheidungen haben somit unverhältnismäßig großen Einfluss auf das Gesamtbild. Die jüngsten Zahlen von Invest Europe zeigen auch, dass in den Jahren 2016 und 2017 diese Schwankungen wiederum "nach unten" ausschlagen (Gassler et al., 2018).

Demgegenüber steht eine stetigere Entwicklung hinsichtlich der Beteiligungsfälle, d.h. der Anzahl von Unternehmensgründungen in denen VC-Fonds investierten. Hier findet sich ein annähernd kontinuierlicher der Risikokapitalmärkte in Europa. Diese Statistiken sind nunmehr leider nur für zahlende Mitglieder zugänglich. Dankenswerterweise hat der Autor exklusiv für eine andere einschlägige Publikation (Gassler et al., 2018) Zugriff auf diese Daten erhalten. Der jeweils aktuelle Rand (bis einschließlich des Jahres 2017) kann somit dort eingesehen werden.

$2 \quad$ Hier wird die Market-Betrachtung herangezogen, d.h. die Investitionen belaufen sich auf die Summe der Investitionen von inländischen und ausländischen VC-Fonds innerhalb Österreichs. VC-Investitionen von österreichischen VC-Fonds im Ausland werden hier also nicht mitgezählt. 
Anstieg von 54 Unternehmen (2007) auf nunmehr 109 im Jahr 2015, was fast exakt einer Verdoppelung entspricht. dennoch sehr deutlich ausgeprägt ist. Nichtsdestotrotz zeigt sich für 2015 eine deutlich bessere Positionierung Österreichs im europäischen

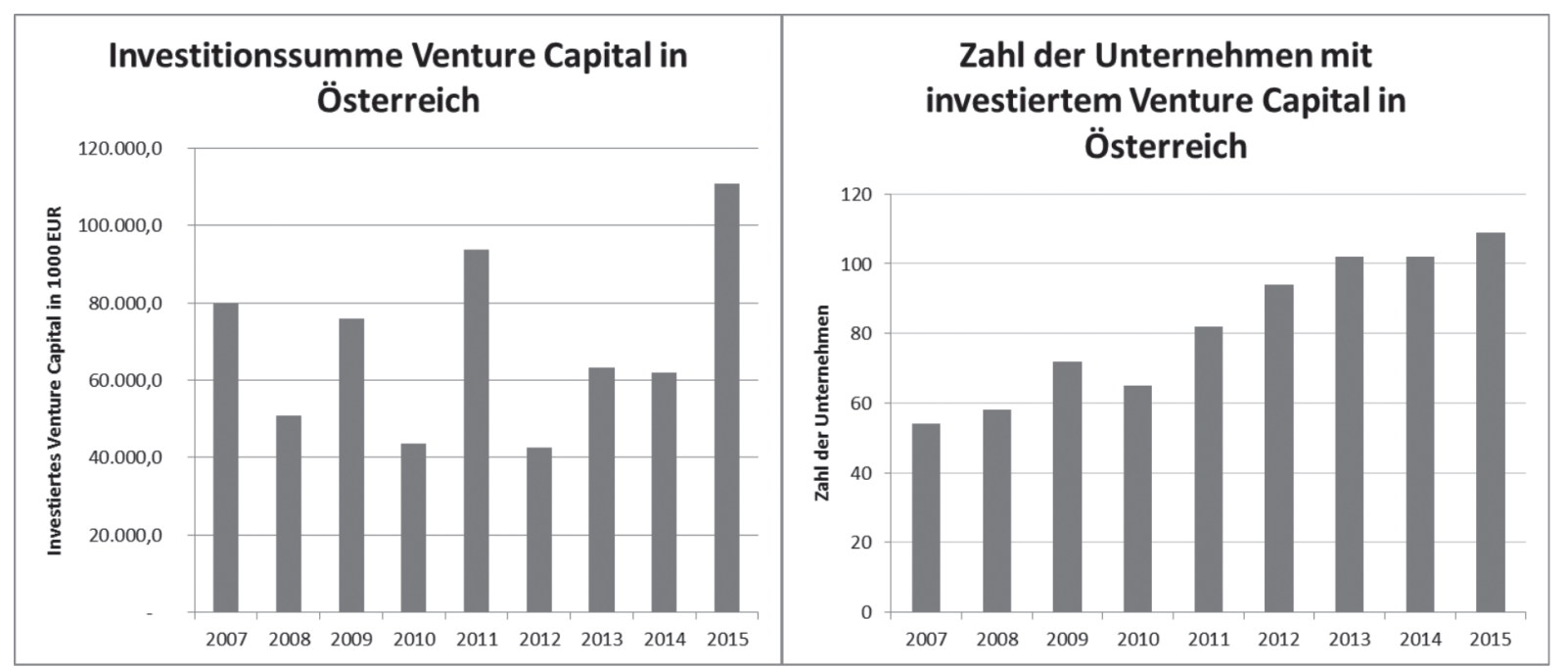

Abbildung 2: Entwicklung der Venture Capital-Aktivitäten (Investitionen und Zahl der Beteiligungsfälle) in Österreich

Quelle: EVCA 2016, eigene Berechnungen, für den aktuellen Rand auf Basis der jüngsten Statistik von Invest Europe siehe Gassler et al., 2018.

In Abbildung 3 wird die internationale (bzw. europäische) Positionierung Österreichs in Bezug auf den Risikokapitalmarkt dargestellt, wobei als Vergleichsgröße der Anteil des Venture Capitals am BIP herangezogen wird ${ }^{3}$. Österreich findet sich diesbezüglich im Jahr 2015 im oberen Mittelfeld der hier betrachteten europäischen Staaten, wobei jedoch der Abstand zu den führenden Ländern wie Finnland und die Schweiz
Vergleich als in der einschlägigen österreichischen Diskussion üblicherweise angenommen wird. Anzumerken ist auch diesbezüglich die Veränderlichkeit im Ranking aufgrund von starken jährlichen Schwankungen. Tatsächlich zeigt sich für den aktuellen Rand (2017), dass Österreich wieder hinter Länder wie Dänemark, Frankreich oder Deutschland zu liegen kommt (siehe Gassler et al, 2018).

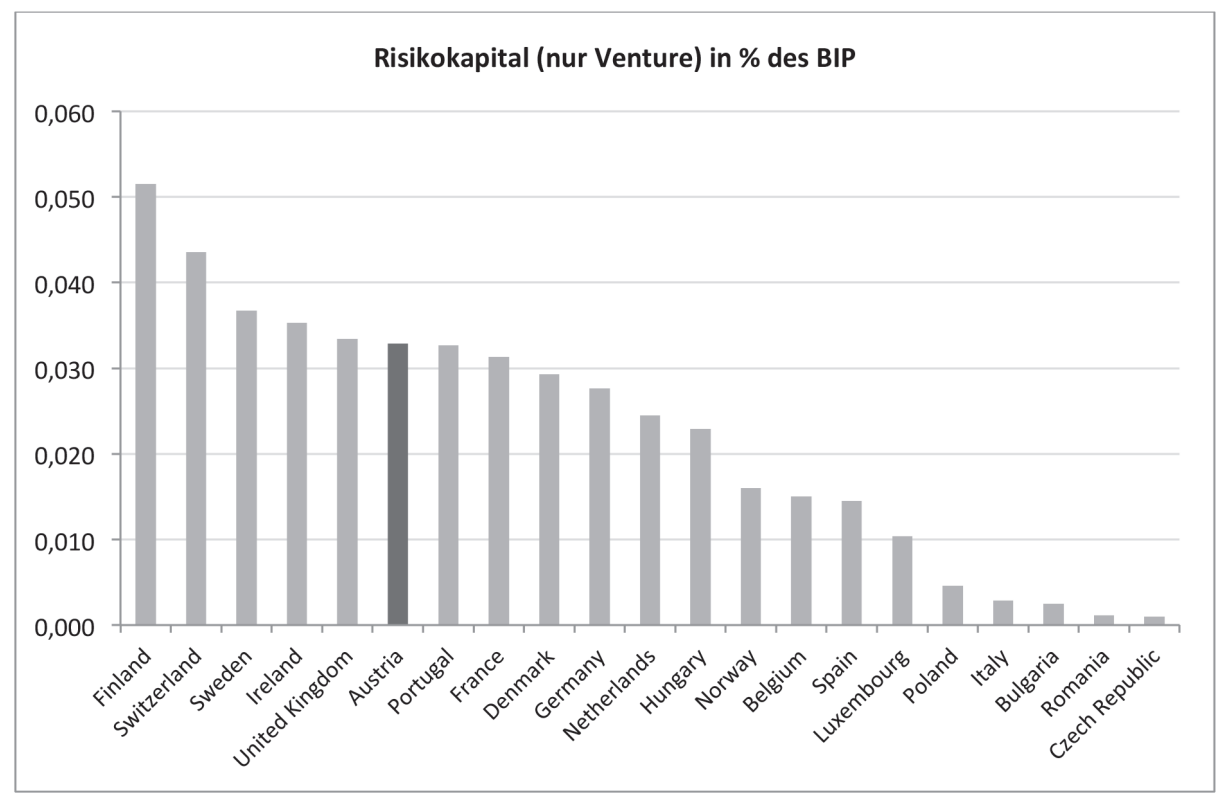

Abbildung 3: Risikokapitalmarkt für Startups im europäischen Vergleich: VC-Investitionen in Relation zum BIP 2015

Quelle: EVCA 2016, eigene Berechnungen, für den aktuellen Rand auf Basis der jüngsten Statistik von Invest Europe siehe Gassler et al., 2018.

3 Hier wird nur das für Unternehmensgründungen relevante Risikokapital - also jenes für die ersten drei Phasen - herangezogen. Risikokapital für die GrowthPhase sowie für andere Typen (z.B. BuyOuts etc.) findet in dieser Betrachtung keine Berücksichtigung. 
In Abbildung 4 wird die zeitliche Entwicklung des Anteils von VCInvestitionen am BIP im internationalen Vergleich für ausgewählte Länder (Österreich sowie die Innovation Leader Länder des European Innovation Scoreboard (kurz EIS)) dargestellt. Die Entwicklung zeigt für Österreich einerseits die bereits bekannte Volatilität in Bezug auf die Risikokapitalinvestitionen, die dazu führt, dass auch in Relation zum BIP die Schwankungsbreite zwischen den Jahren sehr hoch ist (mit einem Minimum wiederum im Jahr 2012 und dem Maximum jüngst im Jahr 2015) und andererseits dennoch einen (leichten) Trend nach oben. In Verein mit den Rückgang in den führenden Ländern (besonders ausgeprägt ist dieser Rückgang in Schweden) - zeigt sich, dass Österreich deutlich aufgeholt und sich der Rückstand zu den Innovation Leader erheblich verringert hat. reiche Einwerbung von VC-Mittel) und quantitativ (Beteiligungsfälle pro Mio. Bevölkerung) nicht zu verstecken braucht!

Gerade der Vergleich mit der Schweiz zeigt, dass bei internationalen Positionierungen der alleinige Blick auf die Investitionssummen nicht ausreicht. Diese ist in der Schweiz sowohl absolut als auch relativ zum BIP wesentlich höher als in Österreich, während hinsichtlich der Anzahl der Gründungen mit VC-Beteiligung Österreich voran liegt. Die Ursache für diese scheinbare Diskrepanz liegt in der jeweiligen Technologiestruktur der Gründungsszene. In der Schweiz ist die Ausrichtung auf Life Sciences deutlich ausgeprägter als in Österreich - nicht zuletzt ist die Schweiz ein global bedeutender Standort für multinationale Pharmaunternehmen. Die durchschnittliche Investitionssumme pro Gründung ist in den Life Sciences aufgrund der spezifischen Bedingungen (mehrfache

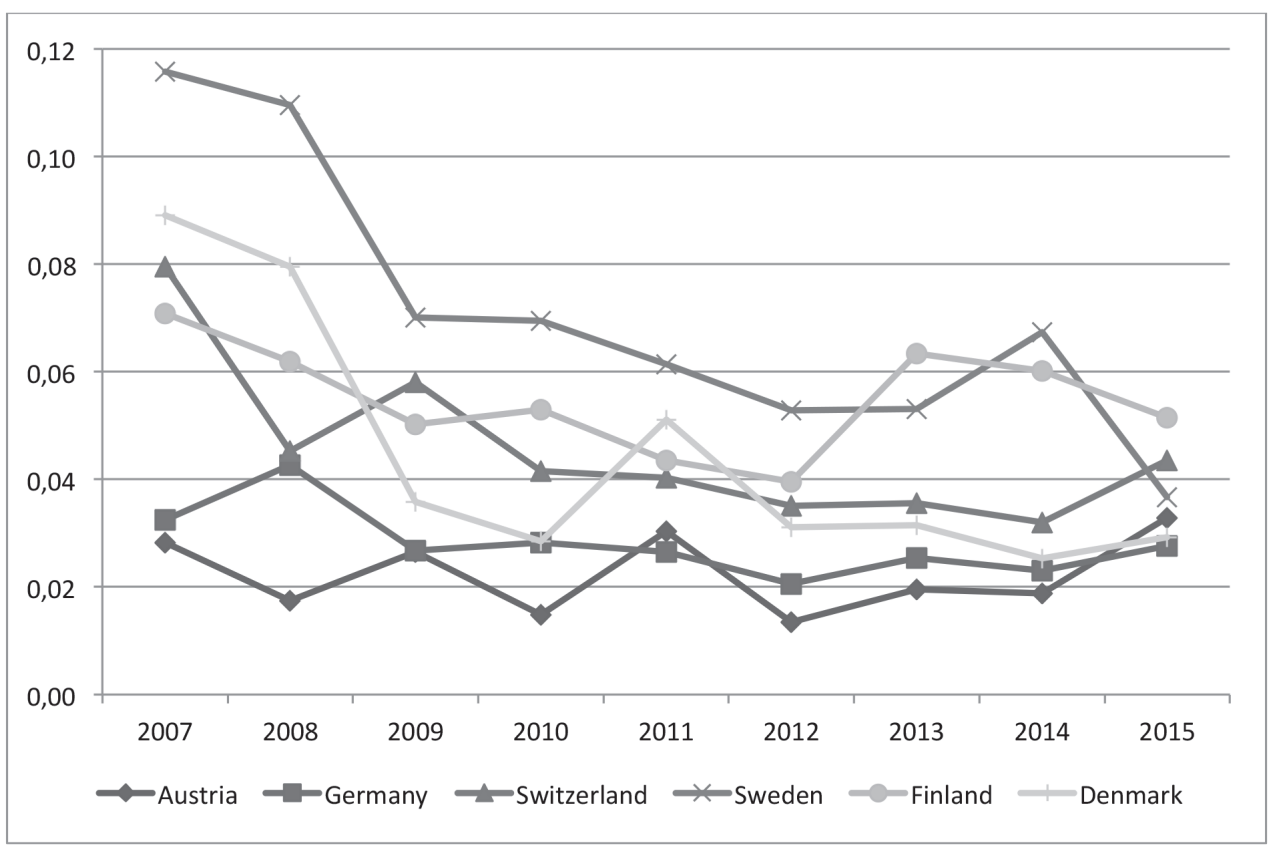

Abbildung 4: Risikokapitalmarkt für Startups im europäischen Vergleich: Entwicklung der VC-Investitionen in Relation zum BIP Quelle: EVCA 2016, eigene Berechnungen

In Abbildung 5 wird die "Dichte" der Venture Capital Aktivitäten gemessen an der Anzahl der Beteiligungsfälle in Portfoliounternehmen 4 im internationalen Vergleich dargestellt. Mit sehr großem Abstand finden sich diesbezüglich die nordischen Staaten Schweden, Finnland und Norwegen (bereits mit Einschränkung) an der Spitze. Österreich liegt bezüglich dieses Indikators immerhin bereits an fünfter Stelle der europäischen Länder! Allerdings ist der Abstand zu den Spitzenländern sehr hoch und der "Vorsprung" Österreichs vor den nachfolgenden Ländern (Niederlande, Deutschland, Dänemark) sehr gering. Dennoch zeigt dieser Vergleich, dass sich Österreichs Gründungsszene qualitativ (erfolg-
Investitionsrunden mit rasant zunehmenden Kapitalbedarf) dieses Technologiefelds mit Abstand höher als in anderen Technologiefeldern, was die Gesamtsumme der VC-Investitionen in der Schweiz ceteris paribus in die Höhe treibt. 


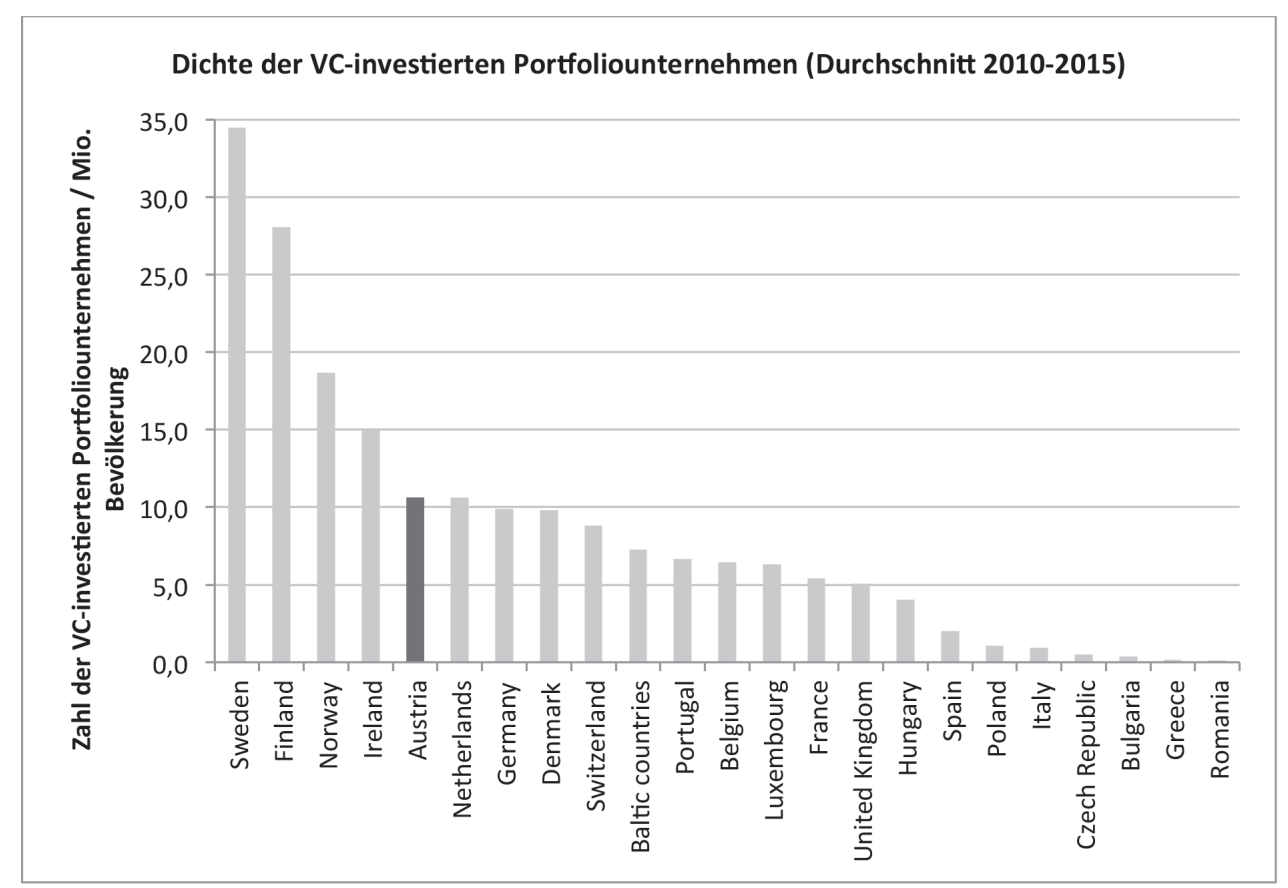

Abbildung 5: Risikokapitalmarkt für Startups im europäischen Vergleich: Zahl der Portfoliounternehmen pro Mio. Bevölkerung

Anm.: Als Bezugsgröße wird hier die Bevölkerung herangezogen, da letztlich Startups üblicherweise von Personen bzw. Personengruppen (Gründerteams) gegründet werden.

Quelle: EVCA 2016, eigene Berechnungen, für den aktuellen Rand auf Basis der jüngsten Statistik von Invest Europe siehe Gassler et al., 2018.

Aus dieser Datenlage und in Verein mit qualitativen Informationen aus der Startup-Szene kristallisieren sich zusammenfassend folgende Tendenzen bzw. Entwicklungslinien bezüglich des österreichischen Risikokapitalmarkts heraus:

- Insgesamt lässt sich feststellen, dass sich die Angebotsseite auf dem Risikokapitalmarkt jüngst deutlich verbessert hat. Die Investitionen in österreichische Gründungen / Startups von Seiten institutioneller VC-Fonds aus dem In- und Ausland lagen im vergangenen Jahr deutlich über den Vergleichswerten des Jahrzehnts davor. Auch die Zahl der VC-Beteiligungen an Gründungen hat sich in Österreich in den vergangenen Jahren positiv entwickelt und ist kontinuierlich angestiegen. Betrachtet man die Zahl der jährlichen VC-Beteiligungen in Relation zur Bevölkerung liegt Österreich mittlerweile im europäischen Vergleich in guter Position (z.B. noch vor Ländern wie Deutschland, Niederlande, Dänemark oder der Schweiz).

- Einige besonders erfolgreiche Exits (d.h. Verkäufe an strategische Partner) haben mittlerweile für eine entsprechend hohe Aufmerksamkeit - auch von Seiten der breiteren Öffentlichkeit - gesorgt. Das Thema Gründen bzw. Startups hat endgültig den medialen Mainstream erreicht.

- Durch diese success stories (erfolgreiche Exits) gelang es den entsprechenden VC-Fonds für ihre nachfolgenden Fondsrunden deutlich leichter und deutlich mehr Investitionsmittel zu akquirieren. Als Beispiel kann hier Speedinvest dienen, deren Fonds Speedinvest II ca. acht- bis neunmal so groß ist wie ihr erster und die eine Vielzahl unterschiedlichster Investoren aus dem In- und Ausland gewinnen konnten.

- Unter diesen Investoren finden sich wiederum auch ursprüngliche Startup-Gründer selbst, die nach entsprechendem wirtschaftlichem Erfolg durch ihr Startup bzw. ihre Startups (Exits) ihre Gewinne in die Gründerszene reinvestieren. Es finden sich somit auch in Österreich mittlerweile eine Reihe von serial entrepeneurs und serial investors (sowie entsprechende Kombinationen aus beiden), die einschlägige track records aufweisen. Insgesamt führt das dazu, dass die Kompetenzen und das Knowhow im System drastisch angestiegen sind. Eine steigende Anzahl an Entscheidungsträgern (Fondsmanager, Business Angels, Serial Entrepreneurs) verfügt über zunehmende Erfahrung. Diese bilden nun die „Knoten“ innerhalb eines - laufend zusätzliches Sozialkapital generierenden - Beziehungsgeflechts, das für potentielle GründerInnen als Knowhow- und Risikokapitalquelle zur Verfügung steht.

- Nach Angaben von Fondsmanagern stellt die Organisation der ersten Finanzierungsrunde (im Bereich von TEUR 100 bis ca. TEUR 300) mittlerweile keinen Flaschenhals in der österreichischen Gründungslandschaft dar. Problematischer wird es erst ab einem größeren Kapitalbedarf von ca. TEUR 500 und mehr. Diese Finanzierungsproblematik betrifft allerdings die jeweiligen Technologiefelder in unterschiedlichem Ausmaß. Vor allem Life Science Gründungen haben diesbezüglich immer noch Schwierigkeiten (die sich in den notwendigen späteren Runden 
hier nochmals deutlich verstärken). Im Segment der IT/Mobile/ Web ist der unmittelbare Kapitalbedarf in der Frühphase nicht mehr so hoch, da vieles auch extern zugekauft werden kann (Serverkosten, Dienstleistungen, Coding etc.).

- Diese angeführten Entwicklungen lassen den Schluss zu, dass das österreichische Startup-Ökosystem mittlerweile an "Reife" gewonnen hat und im Begriff ist, sein Stadium der "infancy" zu verlassen. Es bleibt jedoch noch abzuwarten, wie nachhaltig sich diese skizzierten positiven Tendenzen gestalten und inwiefern das österreichische Startup-Ökosystem bereits in der Lage ist auch eventuelle Rückschläge verkraften kann.

\section{INSTRUMENTE ZUR ANKURBELUNG DES RISIKOKAPITALMARKTES}

Im Jahr 2016 beauftragte das (damalige) BMWFW gemeinsam mit der AWS eine Studie zur Evaluierung der unterschiedlichen eigenkapitalorientierten Fördermaßnahmen der AWS (diese beinhalten die AWS Venture Capital Initiative und die AWS Cleantech Initiative, den AWS
Gründerfonds, den AWS Mittelstandsfonds sowie den AWS Business Angel Fonds). Mit diesen Instrumenten (sowie in Verein mit zusätzlichen gründungsorientierten Förderprogrammen der AWS wie z.B. Preseed und Seedfinancing) deckt die AWS alle relevanten Phasen des Gründungszyklus von Unternehmen ab (siehe Abbildung 6).

Diese Studie wurde unter der Federführung der PWC Austria GmBH und unter Mitarbeit des ZSI im Zeitraum von Mai 2016 bis März 2017 durchgeführt. Da diese Evaluierung vertrauliche Daten auf Individualniveau (z.B. einzelne Fonds, einzelne Business Angels etc.) beinhaltet und daher auch nicht veröffentlicht werden kann, können hier nur allgemeine Aussagen zusammenfassend getätigt werden. In Abbildung 7 werden derartige allgemeine Daten und Ergebnisse der Evaluierung (Basisdatum ist der 31. 3. 2016) der aws Eigenkapitalinstrumente dargestellt. In Österreich konnte im Zeitraum von 2012 bis 2015 sowohl das investierte VC von rund EUR 40 Mio. auf EUR 100 Mio. gesteigert als auch die Anzahl der Beteiligungsunternehmen von rund 100 auf 120 erhöht werden. Die Summe des von der aws investierten Kapitals plus der damit verbundenen Co-Investments anderer (privater) Fonds beträgt zum Stichtag 31. 3. 2016 rund EUR 98 Mio. Insgesamt hielten VCl, aws Gründer- \& Mittelstandsfonds und Business Angel Fonds zum erwähnten Stichtag 66 Beteiligungen. Es wurden bereits 17 Abschichtungen (Exits) realisiert. Die Branchenaufteilung zeigt 13 Beteiligungen im Bereich Life Sciences, $26 \mathrm{im}$ Bereich IT/Web/Mobile und 27 in den restlichen Branchen 5.

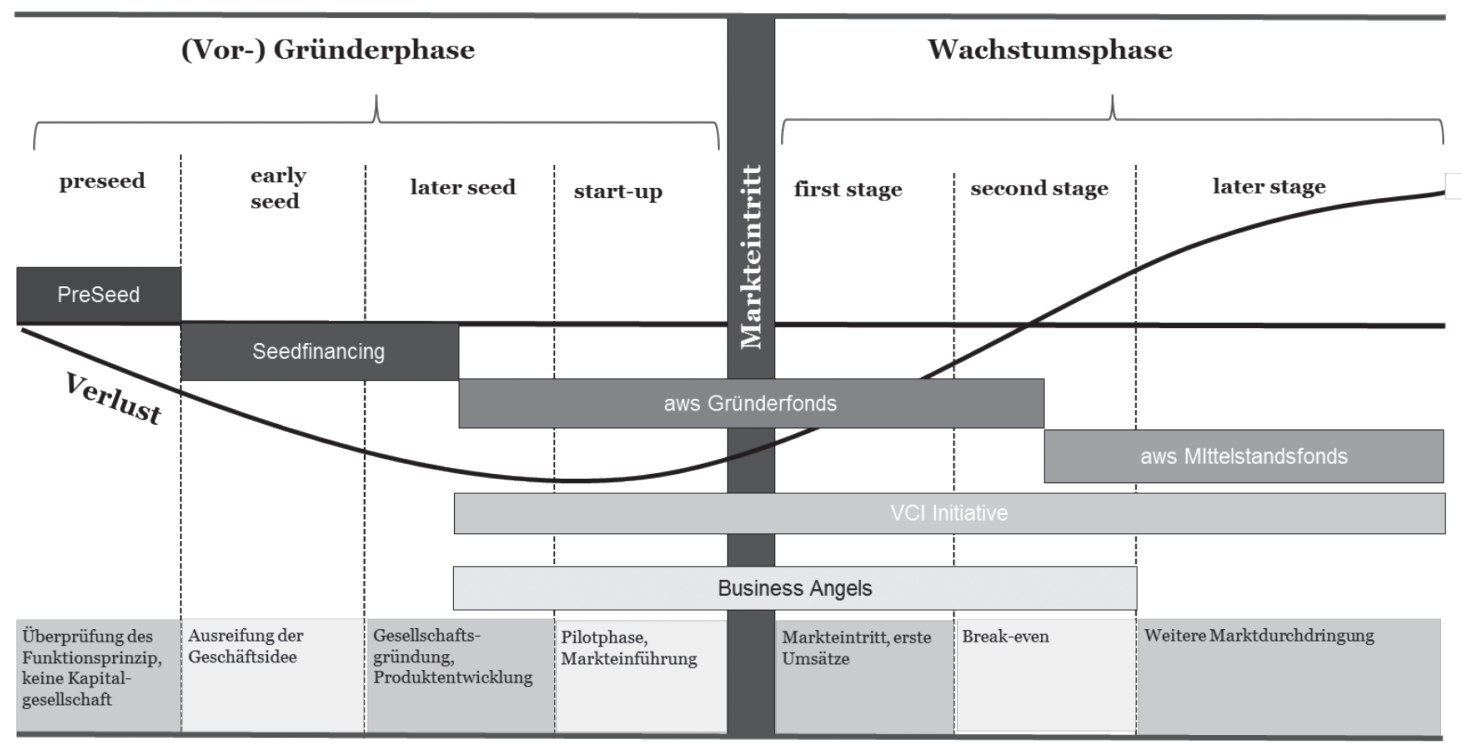

Abbildung 6: Positionierung ausgewählter AWS Förderinstrumente im Gründungszyklus

Quelle: eigene Darstellung 

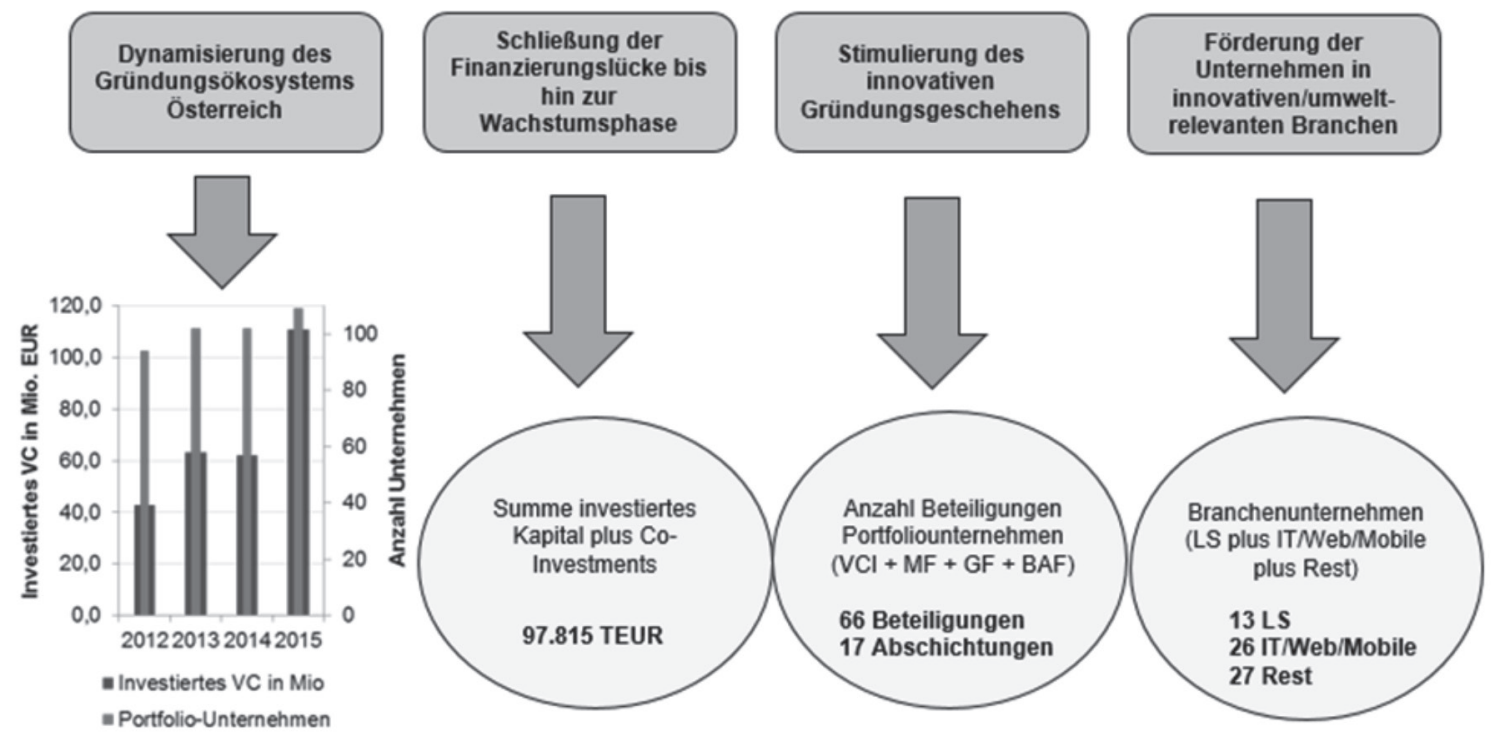
Abbildung 7: Ausgewählte Wirkungen der aws Eigenkapitalinitiativen
Quelle: EVCA 2016, aws

Angesichts der betrachteten Daten und Entwicklungen fällt die zusammenfassende Einschätzung des Risikokapitalmarkts in Österreich je nach betrachtetem Segment bzw. Phase unterschiedlich aus. So ist die Entwicklung des Risikokapitalmarktes in der Seed und- der Startup Phase mittlerweile durchaus positiv zu bewerten. Dies gilt insbesondere für Gründungsprojekte mit geringerem Kapitalbedarf wie sie z.B. im Bereich IT/Web/Mobile zu finden sind. Hier sind (auch nach Aussagen von einschlägigen VC-Investoren) mittlerweile ausreichende Finanzierungsinstrumente und (auch private) Kapitalgeber (Business Angels) vorhanden.

Gleichzeitig ist in diesen Bereichen bereits eine aktive "Szene“ bzw. "Community" entstanden, so dass Information (z.B. über spannende, aussichtsreiche Projekte) rasch übertragen werden. Ausgewählte Business Angels treten vermehrt als "Lead Investors" und "Gatekeepers" auf und bilden die Informationsdrehscheiben und Knoten innerhalb der Community. Oft stellen sie auch wichtige Knowhow-Vermittler für die Gründungsprojekte auf den Weg Richtung formaler Risikokapitaltauglichkeit für klassische VC-Fonds dar. Das erfolgreiche Anlaufen des Business Angel Fonds zeigt, dass das österreichische Gründungsökosystem bereits eine gewisse Reife erreicht hat und es ist bereits abzusehen, dass durch die zusätzlichen Co-Invest-Mittel des Business Angel Fonds entsprechende Effekte in Richtung einer nachhaltigen Dynamisierung erzielt werden.

Teilweise tauchte in Gesprächen mit VC-Fonds bereits die Frage auf, inwieweit es in Österreich überhaupt ein genügend großes Aufkommen an neuen Gründungsprojekten mit potentieller Risikokapitalfähigkeit zur Absorption des investitionswilligen Kapitals gäbe. Dies ist auch vor dem Hintergrund zu betrachten, dass derzeit die Akquise von Investitionskapital für (erfolgreiche) VC-Fonds in Österreich keine Barriere darstellt.
Gerade die Tatsache, dass es mittlerweile eine Serie von erfolgreichen (und medial wahrgenommenen) Exits gibt, hat dazu geführt, dass die Bereitschaft bei potentiellen Investoren einschlägiges Risikokapital zur Verfügung zu stellen derzeit vergleichsweise hoch ist (hilfreich ist dabei natürlich auch, dass die Renditen bei anderen, herkömmlicheren Investitionen immer noch niedrig sind). Gleichzeitig haben die geänderten regulatorischen Rahmenbedingungen auch crowd-investing in Österreich ermöglicht bzw. erleichtert.

Hervorzuheben für diese positive Entwicklung ist die Rolle der AWS und ihrer einschlägigen Instrumente. Im Rahmen der hier analysierten VC-Initiative waren es nicht zuletzt die Investitionen in einen entsprechenden österreichischen VC-Fonds, dessen Erfolg zu zahlreichen dieser erwähnten Success Stories (Exits) geführt hat und somit konnte auch eine zweite Fonds-Runde als attraktives Investitionsvehikel für potentielle (private wie institutionelle) Anleger (darunter auch wieder die awsVC-Initiative) platziert werden. Auch die Aktivitäten des AWS-eigenen VC-Fonds haben entsprechende Impulse auf beiden Seiten des Risikokapitalmarktes gesetzt. Durch Syndizierungen ${ }^{6}$ konnten auch ausländische VC-Fonds an die österreichische Gründungsszene herangeführt werden. Der AWS-Gründerfonds hat aber auch - als kompetenter Ansprechpartner für einschlägige Gründungsprojekte - entsprechenden Einfluss auf den Dealflow ${ }^{\top}$ an interessanten Startup-Projekten für die gesamte österreichische Szene (was letztlich auch an der Anzahl der Co-Investments zu sehen ist). Diese Ausführungen zeigen, wie wichtig die Verzahnung komplementärer wirtschaftspolitischer Instrumente gerade beim Aufbau eines Startup-Ökosystems ist.

Problematischer ist die Situation hingegen jedoch noch bei kapitalintensiveren Anschlussfinanzierungen (etwa ab einem Kapitalbedarf von 
500.000 EUR). Diese sind immer noch schwieriger zu finden und auch für die noch immer recht kleinen rein österreichischen VC-Fonds ist diese Ticketgröße problematisch, da u.U. die Risikostreuung bei derart hohen Ticketgrößen nicht mehr gewährleistet werden kann. Jedenfalls sind bei derartigen Anschlussfinanzierungen internationale Syndizierungen oft notwendig. Dies betrifft insbesondere Startups im Bereich der Life Sciences wo der Finanzierungsbedarf (insbesondere in späteren Runden) deutlich höher ist.

Besonders betont werden soll hier die Wichtigkeit von Dauerhaftigkeit (Nachhaltigkeit) der Instrumente („langer Atem“, vgl. Lerner, 2010), da sie die Planbarkeit für die Entscheidungsträger (Investoren, Gründerlnnen etc.), die sowieso notwendigerweise unter einem hohen Ausmaß an Unsicherheit agieren müssen, zumindest im Bereich dieses wirtschafts- bzw. förderungspolitischen Umfeldes erhöht. Die Entwicklung eines "Startup-Ökosystems" ist ein langfristiger (und langwieriger) Prozess, messbare Ergebnisse lassen u.U. länger auf sich warten und mit Rückschlägen ist durchaus zu rechnen. Es bleibt zu hoffen, dass die österreichische Wirtschaftspolitik diesen "langen Atem" aufweist und sich die momentane "Startup-Begeisterung" nicht als Eintagsfliege erweist.

\section{LITERATUR}

Ács, Z. J., Autio, E. and Szerb, L. (2014): National Systems of Entrepreneurship: Measurement issues and policy implications, Research Policy, 43, 476-494.

Gassler, H., Pointner, W., Ritzberger-Grünwald, D. (2018): Financing growth and innovation in Austrian small and medium sized enterprises, In: OeNB (eds.): Financial Stability Report 36 (in Druck).

Grilli, L. and Murtinu, S. (2014): Government, venture capital and the growth of European high-tech entrepreneurial firms, Research Policy, 43, 1523-1543.

Lerner, J. (2009): Boulevard of Broken Dreams: Why Public Efforts to Boost Entrepreneurship and Venture Capital Have Failed - and What to Do about It, Princeton University Press, Princeton.

Lerner, J. (2010): The future of public efforts to boost entrepreneurship and venture capital, Small Business Economics, 35, 255-264.

OECD (2014): Policy Implications of OECD Experience in Seed and Early Stage Finance, Paris, DSTI/Ind(2014)5.

Schramm, C. (2006): The Entrepreneurial Imperative, Harper Collins Publishers, New York.

\section{AUTOR}

\section{HELMUT GASSLER}

Zentrum für Soziale Innovation

Linke Wienzeile 246

1150 Wien 